\title{
Triiodothyronin, Thyroxine and Thyrotropin in Vitiligo
}

\author{
Subba $K,{ }^{1}$ Karn $D,{ }^{2}$ Khatri $\mathrm{R}^{3}$
}

${ }^{1}$ B\&B Hospital, Gwarko, Lalitpur.

${ }^{2}$ Department of Dermatology and Venereology

Kathmandu University School of Medical Sciences Dhulikhel Hospital, Kavre, Nepal

${ }^{3}$ Patan Academy of Health Sciences, Patan, Lalitpur.

\section{ABSTRACT}

\section{Background}

Vitiligo is a common pigmentary disorder of the skin, affecting individuals globally. Not only is this ailment psychologically incapacitating, it also has a high incidence of autoimmunity, signifying that its manifestations may be the portrayal of dysfunction of immune system.

\section{Objective}

The aim of this study is to find out the situations of abnormal thyroid function test (TFT) in vitiligo patients.

\section{Corresponding Author}

Dr. Kamana Subba

Department of Paediatrics

B\&B Hospital, Lalitpur, Nepal

Email: phuche_001@yahoo.com

\section{Citation}

Subba K, Karn D, Khatri R. Triiodothyronine, thyroxine and thyrotropin in vitiligo. Kathmandu Univ Med J 2011;34(2)7-10.

\section{Methods}

A prospective cross sectional study was conducted at the Dhulikhel Hospital, KUTH, Dhulikhel during November 2008 to January 2011 with the objective to assess the thyroid function tests in vitiligo patients.

\section{Results}

Total 66 vitiligo patients with male 30 (45\%) and female 36 (55\%). Total 26 (39.39\%) vitiligo patients have been found to have abnormal thyroid function test. Among them 10 had abnormal T3, seven had abnormal T4 and 12 had abnormal TSH level. Thyroid function test were normal in other vitiligo patients. Total seven vitiligo had high level of T3 than normal value, four male and three female. Two had higher level of T4 above 2.0 $\mathrm{ng} / \mathrm{dl}$ and all were male, while five had abnormally low level of T4 with three male and two female. There were seven vitiligo patient with increased T3 level and three with decreased T3 level, among them six were males and four were females, the age group was ranged from seven to 68 years old. The T4 level were found abnormal in seven vitiligo patients among which five were males and two were females with the age group ranging from 7 to 51 years.

\section{Conclusions}

There has been significant association of thyroid disorder in the patient with vitiligo. Therefore, patient with vitiligo need to undergo thyroid function test to rule out the thyroid disorder and prevent from long-term complications.

\section{KEYWORDS}

thyroid, thyroxin, thyroid function test, vitiligo

\section{INTRODUCTION}

Vitiligo is an acquired skin disorder caused by the disappearance of pigment cells from the epidermis that gives rise to well-defined white patches, which are often symmetrically distributed. ${ }^{1}$ Vitiligo occurs worldwide with an overall prevalence of $1 \%$. However, its incidence ranges from 0.1 to $8.8 \%$ across India and in different countries of the globe. ${ }^{2}$ The autoimmune theory proposes that abnormalities of the immune system result in destruction of melanocytes. This hypothesis is initially supported by the frequent observation that several autoimmune disorders (thyroid disorders, juvenile diabetes mellitus, and Addison's disease, pernicious anemia) are associated with vitiligo.

Vitiligo is associated with a large variety of diseases like thyroid disorders, pernicious anaemia, diabetes mellitus, alopecia areata, morphoea, lichen sclerosus, lupus erythematosus, halo nevus, malignant melanoma, rheumatoid arthritis, ulcerative colitis etc. ${ }^{2}$ The association 
of vitiligo and thyroid diseases assumes significance as the incidence of thyroid disease in vitiligo is reported to vary from $0.62-12.5 \%$, approaching even $39 \%$ in elderly patients. A common pathogenesis for the two conditions is further suggested by the reported effect of decreased cutaneous oxidation in causing depigmentation in rabbits and chickens by injections of thyroid extract and thyroxine; development of diffuse goiter, increased TSH secretion, hepatosplenomegaly and vitiliginous lesions in a group of factory workers exposed to para-tertiary-butylphenol; increased levels of antithyroglobulin antibodies in vitiligo and association of these two with other disorders of autoimmune origin. ${ }^{3-6}$

\section{OBJECTIVE}

The objective of this study was to find out the prevalence of thyroid disorder in patient with vitiligo.

\section{METHODS}

A prospective observational study was conducted in Department of Dermatology, Dhulikhel Hospital Kathmandu University Hospital from November 2008 to January, 2011. The patients with vitiligo attending OPD of Dhulikhel hospital were included in the study and the diagnosis of vitiligo in all cases was made based on the clinical features alone. Those patients who could afford the test were included in the sample after informed consent.

Blood samples were drawn after an overnight fast. fT3, fT4 and TSH were assayed in each sample using radio-immuneassay (Eliscan, product of RFCL, India) and the results were expressed in terms of $\mathrm{pg} / \mathrm{ml}, \mathrm{ng} / \mathrm{dl}$ and micro international units $/ \mathrm{ml}$ respectively. The data entry and statistical analysis were done using Microsoft Excel 2010 in statistical package for social sciences (SPSS) version 14 for windows.

\section{RESULTS}

There were total 66 vitiligo patients with male $30(45 \%)$ and female 36 (55\%). Total 26 (39.39\%) vitiligo patients have been found to have abnormal thyroid function test (Figure 1). Among them 10 had abnormal T3, seven had abnormal T4 and 12 had abnormal TSH level (Table 1, Figure 1). Similarly thyroid function test were normal in other vitiligo patients (Table 2, Figure 2).

Table 1. Abnormal Level of TFT in patient with vitiligo.

\begin{tabular}{|llll|}
\hline TFT level & Male & Female & Total \\
\hline T3 & $6(60 \%)$ & $4(40 \%)$ & 10 \\
\hline T4 & $5(71 \%)$ & $2(29 \%)$ & 7 \\
\hline TSH & $3(25 \%)$ & $9(75 \%)$ & 12 \\
\hline
\end{tabular}

Table 2. Normal Level of TFT in vitiligo patients.

\begin{tabular}{|l|l|l|l|}
\hline TFT level & Male & Female & Total \\
T3 & $24(43 \%)$ & $32(57 \%)$ & 56 \\
\hline T4 & $25(42 \%)$ & $34(58 \%)$ & 59 \\
TSH & $27(50 \%)$ & $27(50 \%)$ & 54 \\
\hline
\end{tabular}

Total 7 vitiligo had high level of T3 than normal value. Among them four were male and three were female. Two had higher level of T4 above $2.0 \mathrm{ng} / \mathrm{dl}$ and all were male, while five had abnormally low level of T4 with three male and two female.

There were seven vitiligo patient with increased and three with decreased T3 level, among them six were males and four were females, the age group was ranged from seven to 68 years old.

The T4 level were found abnormal in seven vitiligo patients among which five were males and two were females with the age group ranging from seven to 51 years.

Total 12 patients with vitiligo had abnormal TSH level among which three were males and nine were females with the age group ranging from seven to 51 years.

\section{DISCUSSION}

Vitiligo is an acquired skin depigmentation that affects all human races. The precise cause of vitiligo is unknown. ${ }^{7}$ Genetic factors appear to play a role; 20 to 30 percent of patients may have a family history of the disorder. The pathogenesis is thought to involve an autoimmune process directed against melanocytes. Evidence of this is derived from histologic studies that have found an absence of melanocytes in the affected skin. ${ }^{8}$ An autoimmune process is also suggested by the concomitant occurrence of other autoimmune diseases in patients with vitiligo. Alterations in both cellular and humoral immunity have been reported. Evidence supports cellular immunity as an important factor in the development of vitiligo. CD8+ T cell predominant infiltrates have been found in perilesional skin, and CD8+ cytotoxic $T$ cells have been identified adjacent to disappearing melanocytes. ${ }^{9}$ Multiple autoantibodies against melanocyte antigens including tyrosinase, tyrosinase related proteins 1 and 2, Pmel17, SOX transcription factors, and melanin-concentrating hormone receptor 1 (MCHR1) have been detected in the sera of some patients with vitiligo. ${ }^{9}$ Whether these autoantibodies play a significant role in the pathogenesis of the disorder remains unclear. Cytokines may play an important role in the pathogenesis of vitiligo. There has been increased frequencies of other autoimmune disorders, such as autoimmune thyroid disease, pernicious anemia, systemic lupus erythematosus, and Addison's disease have been detected in patients with vitiligo. ${ }^{10}$ The association of vitiligo with these disorders suggests the presence of shared genetic factors that 
contribute to the development of these diseases. In a genome wide association study, several genes with known associations with other autoimmune disorders were identified as potential susceptibility loci for generalized vitiligo, including PTPN22, LPP, IL2RA, UBASH3A, C1QTNF6, and genes encoding $\mathrm{MHCI}$ and $\mathrm{MHC} \mathrm{II} \mathrm{molecules.}{ }^{11}$

In our study we found 26 (39.39\%) vitiligo patients with abnormal thyroid function test from total 66 vitiligo patients. Among them ten had abnormal T3, seven had abnormal T4 and 12 had abnormal TSH level. A study done by Nishi Arora VK et al have shown that six cases comprising four males and two females showed higher T3 values. All of them had generalized vitiligo. T4 and TSH levels were normal in all the cases contrary to which our study shown, seven abnormal T4 and 12 abnormal TSH level. In their control group, the respective values for T3, T4 and TSH were within the normal ranges of 70 to $220 \mathrm{ng} / \mathrm{dl}, 4.7$ to $11.0 \mu \mathrm{g} /$ $\mathrm{dl}$ and 0-10 units/ $\mathrm{ml}^{2}$ In their study, none of the patients had clinical evidence of thyroid disorder. Still, higher values for T3 were recorded in six patients who otherwise had a normal level of thyroxine and thyrotropin. This condition has been termed "T3-toxicosis" and it can occur either due to a mild or early hyperthyroidism, or during treatment of hyperthyroid state. ${ }^{12}$ But in our case we found abnormal level of T3 in ten patients. It indicates they have some level of thyroid disorder.

Since none of our patients was on any drug therapy, it must be a mild adaptive change towards hyperthyroid state. On the other hand, a mild hypothyroid state due to a non-thyroidal illness leads to lowering of T3 values although these are within the normal range. ${ }^{13} \mathrm{TSH}$ values are considered to be the best indicator of thyroid disease in vitiligo. And in our cases we had 12 cases of abnormal TSH level in the vitiligo patients. This also indicates presence of thyroid disorder among them. But only, five patients showed clinical symptoms of thyroid disorders. May be despite of abnormal TSH in 12 patients, rest of them were asymptomatic.

However in study done by Nishi Arora VK et al, none of their cases showed any derangement of TSH level, it confirms their clinically euthyroid status. ${ }^{2}$ Therefore, the changes in other thyroid hormone levels in vitiligo are merely an adaptive response to non-thyroidal illness. However, it is necessary to follow such patients for a longer period to look for the development of overt thyroid dysfunction and also to note whether these adaptive changes are reversed on successful treatment of vitiligo.

A clinical study of one hundred patients having vitiligo done by Martis et al revealed the incidence among new patients to be $1.84 \%$. The male/female ratio was $1: 1.22$. Family history of vitiligo was available in $8 \%$ of our patients.
The different morphological pattern consisted of vitiligo vulgaris, focal vitiligo, acrofacial vitiligo, lip-tip vitiligo, lip vitiligo, segmental vitiligo and universal vitiligo. Associated diseases include atopic dermatitis, Hansen's disease, alopecia areata, halo naevus, chronic urticaria, lichen planus, diabetes mellitus, hypertension, hypothyroidism, epilepsy and IHD. ${ }^{14}$ However in our cases we didn't categorized vitiligo per se nor do we rule out any other disorder, this limitation can be addressed by more robust study in the future.

Other studies have reported the association of vitiligo with Numerous cutaneous and systemic disorders like diabetes mellitus. ${ }^{15,16}$ In some of these conditions, such as the autoimmune diseases, the incidence of vitiligo is significantly higher than in the population at large. This study was done in small population in a center, a robust study with community sampling would reveal the true nature of thyroid disorder in vitiligo patients.

\section{CONCLUSION}

There has been association of thyroid disorder in the patient with vitiligo. Therefore, patient with vitiligo need to undergo thyroid function test to rule out the thyroid disorder and prevent from long-term complications. A long-term follow up is required with vitiligo patient wit thyroid disorder and timely intervention is needed.

\section{REFERENCES}

1. Njoo MD, Westerhof W. Vitiligo. Pathogenesis and treatment. Am J Clin Dermatol. 2001;2(3):167-81.

2. Nishi Arora VK, Shankar V, Chaudhary SD, Nagpal RK. Triiodothyronine, thyroxine and thyrotropin levels in vitiligo. Indian J Dermatol Venereol Leprol. 1990;56(4):299-300.

3. Peracchi M, Cavagnini F, Ghislanzoni G. [Anti-thyroid antibodies and autoimmune diseases in patients with vitiligo]. G Ital Dermatol Minerva Dermatol. 1971 Nov;46(11):487-91.

4. Daneshpazhooh M, Mostofizadeh GM, Behjati J, Akhyani M, Robati RM. Anti-thyroid peroxidase antibody and vitiligo: a controlled study. BMC Dermatol. 2006;6:3.

5. Zettinig G, Tanew A, Fischer G, Mayr W, Dudczak R, Weissel M Autoimmune diseases in vitiligo: do anti-nuclear antibodies decrease thyroid volume? Clin Exp Immunol. 2003 Feb;131(2):347-54.

6. Kemp EH, Gawkrodger DJ, MacNeil S, Watson PF, Weetman AP. Detection of tyrosinase autoantibodies in patients with vitiligo using 35S-labeled recombinant human tyrosinase in a radioimmunoassay. $J$ Invest Dermatol. 1997 Jul;109(1):69-73.

7. Grimes PE. New insights and new therapies in vitiligo. JAMA. 2005 Feb 9;293(6):730-5.

8. van den Wijngaard R, Wankowicz-Kalinska A, Le Poole C, Tigges B, Westerhof W, Das P. Local immune response in skin of generalized vitiligo patients. Destruction of melanocytes is associated with the prominent presence of CLA+ T cells at the perilesional site. Lab Invest. 2000 Aug;80(8):1299-309.

9. Ongenae K, Van Geel N, Naeyaert JM. Evidence for an autoimmune pathogenesis of vitiligo. Pigment Cell Res. 2003 Apr;16(2):90-100. 
10. Alkhateeb A, Fain PR, Thody A, Bennett DC, Spritz RA. Epidemiology of vitiligo and associated autoimmune diseases in Caucasian probands and their families. Pigment Cell Res. 2003 Jun;16(3):208-14.

11. Jin Y, Birlea SA, Fain PR, Gowan K, Riccardi SL, Holland PJ, et al. Variant of TYR and autoimmunity susceptibility loci in generalized vitiligo. $\mathrm{N}$ Engl J Med. 2010 May 6;362(18):1686-97.

12. Kaplan MM. Clinical and laboratory assessment of thyroid abnormalities. Med Clin North Am. 1985 Sep;69(5):863-80.

13. Bermudez F, Surks MI, Oppenheimer JH. High incidence of decreased serum triiodothyronine concentration in patients with nonthyroidal disease. J Clin Endocrinol Metab. 1975 Jul;41(1):27-40.
14. Martis J, Bhat R, Nandakishore B, Shetty JN. A clinical study of vitiligo. Indian J Dermatol Venereol Leprol. 2002 Mar-Apr;68(2):92-3.

15. Gould IM, Gray RS, Urbaniak SJ, Elton RA, Duncan LJ. Vitiligo in diabetes mellitus. Br J Dermatol. 1985 Aug;113(2):153-5.

16. Macaron C, Winter RJ, Traisman HS, Kahan BD, Lasser AE, Green OC. Vitiligo and juvenile diabetes mellitus. Arch Dermatol. 1977 Nov;113(11):1515-7. 